Article

\title{
Structural Stability of Optofluidic Nanostructures in Flow-Through Operation
}

\author{
Yazan Bdour ${ }^{1,+}$, Juan Gomez-Cruz ${ }^{1,2,+(1)}$ and Carlos Escobedo ${ }^{1, *(1)}$ \\ 1 Department of Chemical Engineering, Queen's University, Kingston, ON K7L 3N6, Canada; \\ 16yb6@queensu.ca (Y.B.); 17jmgc@queensu.ca (J.G.-C.) \\ 2 Instituto de Ciencias Aplicadas y Tecnología (ICAT), Universidad Nacional Autónoma de México (UNAM), \\ Ciudad de México 04510, Mexico \\ * Correspondence: ce32@queensu.ca \\ + These authors contributed equally to work.
}

Received: 25 February 2020; Accepted: 31 March 2020; Published: 2 April 2020

check for updates

\begin{abstract}
Optofluidic sensors based on periodic arrays of subwavelength apertures that support surface plasmon resonance can be employed as both optical sensors and nanofluidic structures. In flow-through operation, the nanoapertures experience pressure differences across the substrate in which they are fabricated, which imposes the risk for structural failure. This work presents an investigation of the deflection and structural stability of nanohole array-based optofluidic sensors operating in flow-through mode. The analysis was approached using experiments, simulations via finite element method, and established theoretical models. The results depict that certain areas of the sensor deflect under pressure, with some regions suffering high mechanical stress. The offset in the deflection values between theoretical models and actual experimental values is overturned when only the effective area of the substrate, of $450 \mu \mathrm{m}$, is considered. Experimental, theoretical, and simulation results suggest that the periodic nanostructures can safely operate under trans-membrane pressures of up to $20 \mathrm{psi}$, which induce deflections of up to $\sim 20 \mu \mathrm{m}$.
\end{abstract}

Keywords: optofluidic; sensor; surface plasmon resonance; nanohole array; mechanical properties; nanofluidic; nanoplasmonic

\section{Introduction}

The development of new point-of-care (POC) diagnostic technologies requires low-cost, fully integrated sensing platforms capable of providing quantitative results in situ. At the same time, POC diagnostic platforms have a tremendous potential that is yet to be fully exploited. Telemedicine, for instance, aims to monitor the health of patients remotely through on-site sensing using personal devices, holding a global market of ca. \$20 billion USD (United States dollars) [1]. A trendy and increasingly demanded approach to in situ sensing is the use of lab-on-a-chip platforms enabled by cell phones to record, analyze, and transmit the results [2-4]. With the recent emergence of new pathogens, such as the Coronavirus and the Yaravirus, an on-site analysis will limit their health impact with a rapid sensing test, quantifying the severity of the infection, and assisting with the quarantine measures $[5,6]$. Periodic arrays of subwavelength structures fabricated in metal films enable surface plasmon resonance (SPR), which motivated their use as biosensors for several applications in different fields [7-12]. Ordered arrays of metallic nanoholes are optofludic structures that enable transport of both fluid and analyte via nanofluidic confinement and nanoplasmonic sensor. The plasmonic resonance signature obtained from nanohole arrays (NHAs) allows the detection of biologically relevant analytes in label-free fashion and real time. Toward the development of POC biosensing platforms, these optofluidic nanostructures are integrated into microfluidic environments in order 
to create fully integrated sensors compatible with portable electronics [13]. NHA-based sensors are ideal for field applications due to their small footprint and integration abilities as evidenced by recent demonstrations for the detection of bacteria, such as Chlamydia trachomatis [14], viruses, such as Ebola [15], cancer biomarkers [16] and uropathogenic bacteria [17]. Flow-through optofluidic structures also enable the enrichment of analytes in liquids by an electrohydrodynamic effect occurring around the NHAs when an electric potential and a pressure bias are applied to the fluid in a closed system [18]. Despite their demonstrated potential in sensing, most applications involving nanohole arrays focus on exploiting the conventional optical capacities of these nanostructures. The mechanical stability of the nanohole membranes is an overlooked aspect of their properties that are key when functioning as nanofluidic structures. In analogy to porous silicon-based membranes, where permeability increases significantly as membrane thickness decreases, the volumetric flow across nanostructured optofluidic sensors increases with the open pore fraction. However, plasmonic nanostructures with built-in thin membranes may suffer from low mechanical stability which could limit, critically, their use as optofluidic flow-through sensors [19-21]. The membrane's mechanical properties change due to the change in the structural morphology of the porous membrane as it deflects under pressure. The stability decreases by a correction factor $(1-P)$, where $P$ is related to the porosity of the membrane [22].

Recent studies demonstrate that through-nanoapertures fabricated in thin $(\sim 50 \mathrm{~nm})$ gold-coated $\mathrm{Si}_{3} \mathrm{~N}_{4}$ substrates offer additional fluidic abilities that can be used to target in-hole delivery of analytes when operated as optofluidic sensors [23,24]. However, flow-through operation results in transmembrane pressures that could potentially damage the rather brittle nanostructures. The mechanical properties of the organized nanohole arrays are not completely understood due to their sensitivity, brittleness, and nano-sized structures. Here, we present a study on structural aspects of Au-on-nitride optofluidic nanoplasmonic sensors operating in flow-through fashion at flowrates compatible with biosensing applications.

\section{Materials and Methods}

\subsection{Fabrication of Periodic through Subwavelength Apertures}

Through-nanohole arrays were fabricated using focused ion beam (FIB) milling using 100-nm-thick $\mathrm{Si}_{3} \mathrm{~N}_{4}$ free-standing membranes (Norcada, Edmonton, $\mathrm{AB}$, Canada) coated with a thermally evaporated $100 \mathrm{~nm}$ layer of gold via a 5-nm chromium adhesion layer. Milling was achieved using a gallium ion beam set at $40 \mathrm{keV}$ with a beam current of $\sim 30 \mathrm{pA}$, with a typical beam spot size of $10 \mathrm{~nm}$, and the dwell time of the beam at one pixel was set to $20 \mu \mathrm{s}$. Two arrays of through-nanohole arrays with an area of $20 \mu \mathrm{m}$ by $20 \mu \mathrm{m}$, diameter of ca. $230 \mathrm{~nm}$, and pitch of $560 \mathrm{~nm}$ were fabricated.

\subsection{Fabrication of Microfluidic Chips}

The microfluidic chip was fabricated using a replica molding technique as described in detail elsewhere [25]. The general steps of the fabrication procedure are briefly described next. A mask with the microfluidic pattern was generated using SolidWorks CAD software (Dassault Systems Solidworks Corp., Waltham, MA, USA). The design included one inlet and one outlet of $1.5 \mathrm{~mm}$, and a 5-mm-wide channel with $100 \mu \mathrm{m}$ in height. A master was fabricated by spin-coating SU-8 100 photoresist (MicroChem Corp., Newton, MA, USA) on a clean three-inch silicon wafer (Silicon Quest International Inc., Santa Clara, CA, USA). The coated wafer was then prebaked for one minute at $65^{\circ} \mathrm{C}$ and for $10 \mathrm{~min}$ at $95^{\circ} \mathrm{C}$. The mask with the channel pattern was then placed over the coated wafer and exposed to ultraviolet (UV) light for $90 \mathrm{~s}$. Next, the exposed wafer was hard-baked at $65^{\circ} \mathrm{C}$ for $1 \mathrm{~min}$ and at $95^{\circ} \mathrm{C}$ for $10 \mathrm{~min}$. The master was subsequently developed using a SU-8 developer (MicroChem Corp., Newton, MA, USA). A 12:1 mixture of Sylgard 184 elastomer to curing agent (Dow Corning, Midland, MI, USA) was mixed, degassed in a vacuum, and poured onto the master. After baking at $85{ }^{\circ} \mathrm{C}$ for $20 \mathrm{~min}$, the replica was removed from the mold. Inlets and outlets were provided 1-mm punched holes for fluidic access. Microfluidic connections were achieved using polyether ether ketone 
(PEEK) tubing (Upchurch Scientific, Oak Harbor, WA, USA). A schematic representation of the set-up is shown in Figure 1.
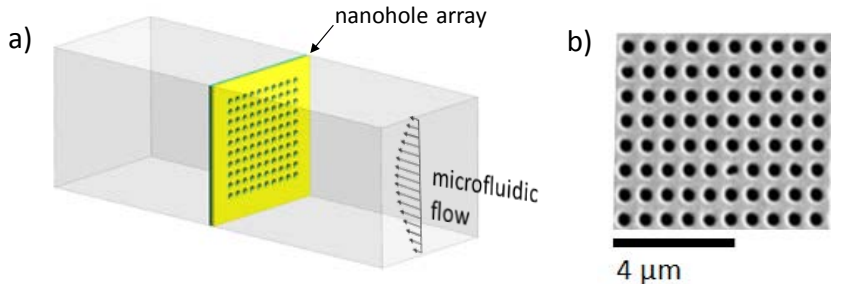

Figure 1. (a) SEM image of fabricated periodic subwavelength apertures via focused ion beam (FIB). The nanostructures were $230 \mathrm{~nm}$ in diameter and $560 \mathrm{~nm}$ in pitch. (b) Schematic representation of a nanohole array in a microfluidic chip in flow-through operation.

\subsection{Optofluidic Structure Deflection Analysis}

Finite element analysis (FEA) was used as a means to know the order of magnitude of the deflection and the mechanical stress that the optofluidic sensor may experience in flow-through operation. COMSOL Multiphysics (COMSOL, Stockholm, Sweden) was used to simulate a simplified model of the optofluidic sensor under a prescribed unidirectional and orthogonal pressure on one of the faces of the suspended membrane. The simulations were used firstly to estimate the order of magnitude of applied pressures that would result on the deflection of the substrate containing the optofluidic structures. This first model involved a stationary elastic model with default Lagrange-quadratic element type. The finite element analysis solves for the displacement field at a specific point on the membrane for every input force. For the linear model, the system is governed by three tensor partial differential equations: $\nabla \cdot \sigma+F_{v}=0, \varepsilon=\frac{1}{2}\left[(\nabla u)^{T}+\nabla u+(\nabla u)^{T} \nabla u\right]$, and $C=C(E, v)$, where $\sigma$ is the Cauchy stress tensor, $F_{v}$ is the body force per unit, $u$ is the displacement vector, $\varepsilon$ is the infinitesimal strain tensor, $C$ is the fourth-order stiffness tensor, $E$ is the Young's modulus, and $v$ is the Poisson's ratio. A second static, nonlinear stress-strain model was used to compare the experimental data and to validate the deflection values obtained for the prescribed pressure range. The nonlinear stress-strain behavior was achieved by using a power-law nonlinear elastic material model, accounting for geometric nonlinearities, which is governed by Ludwik's law, $\tau=\tau_{0}+k \gamma^{1 / n}$, where $\tau$ is the shear stress, $\gamma$ is the shear strain, and $n$ is an integer [26,27]. A user-controlled mesh with Lagrange-quadratic element type was used for this nonlinear model, to guarantee an acceptable mesh size along the thickness of the modeled substrate. The finite element analysis solves for the displacement field at a specific point on the membrane for every input force. In both models, linear and nonlinear, the parameters of $\mathrm{Si}_{3} \mathrm{~N}_{4}$ were mainly used, as the values for the mechanical properties for this material supersede those of the metal components in the sensor, namely, a Young's modulus of $250 \times 10^{9} \mathrm{~Pa}$, a density of $3.1 \times 10^{3} \mathrm{~kg} / \mathrm{m}^{3}$, and a Poisson's ratio of 0.23 . The surrounding surfaces around the membrane that correspond to the areas that define the thickness of the substrate were set as fixed boundaries. The transmembrane pressures were varied from 1 to $20 \mathrm{psi}$, as this range corresponds to flow rates on the order of $\mathrm{nL} / \mathrm{min}$, which are commonly used in biosensing applications. The deflection of the substrate and the stress (von Mises criterion) were recorded.

In addition to finite element method (FEM)-based models, analytical models on the mechanical behavior of perforated membranes published in the literature were also used to estimate the deflection of the optofluidic sensors in this study, as detailed in the Section 3 [22].

\section{Results and Discussion}

Figure 2 shows a schematic representation of the experimental setup used to measure the deflection of the membranes. Figure 3 shows the computer-aided design (CAD) models used to study the deflection of the optofluidic sensors via COMSOL Multiphysics software. Figure 3a shows the simplified model with a single nanoaperture at the center, used in the linear elastic material 
simulations. The model accounts for a 100-nm-thick membrane with a square surface with a side length of $500 \mu \mathrm{m}$, and a circular opening of $10 \mu \mathrm{m}$ for surface coverage equivalency of the effective surface of the nanoapertures. Figure $3 \mathrm{~b}$ shows an image of the CAD model used for the nonlinear simulations, a square 100-nm-thick membrane with side length of $500 \mu \mathrm{m}$ and a $20 \mu \mathrm{m} \times 20 \mu \mathrm{m}$ array of 230-nm-diameter holes with pitch-to-diameter ratio of 2 . In both cases, linear and nonlinear models, the mesh curvature factor was 0.6 , the maximum element scaling factor was 1.9 , the resolution of narrow regions was 0.3 , and the optimize quality feature was set to on. The linear model had a maximum element size at all boundaries of $30 \times 10^{-9}$. The resulting mesh had $\sim 210 \times 10^{3}$ domain elements with $\sim 40 \times 10^{3}$ boundary elements and $\sim 1.4 \times 10^{3}$ edge elements. The nonlinear model had $\sim 1.4 \times 10^{4}$ domain elements, $\sim 900 \times 10^{3}$ boundary elements, and $\sim 6 \times 10^{3}$ edge elements. The models were solved for pressures applied to the bottom surface of the substrate, for $1 \mathrm{psi}$, and then using the sweep parameter feature for a pressure range of 2-20 psi with 2-psi pressure increments.

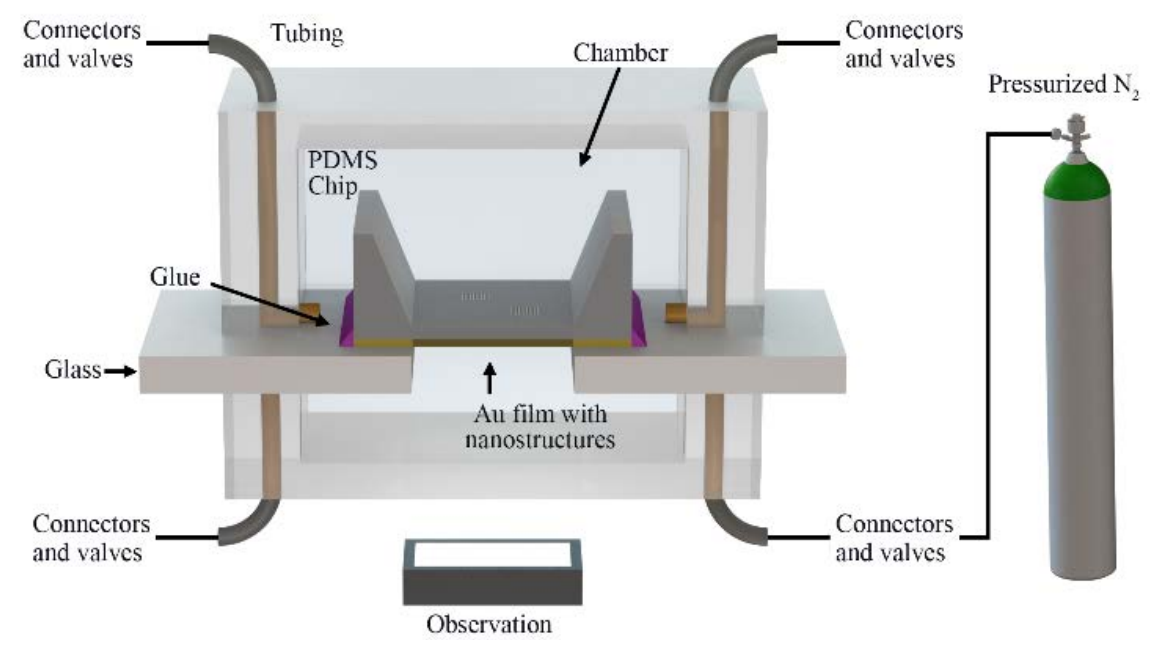

Figure 2. Schematic representation of the experimental set-up.

a)

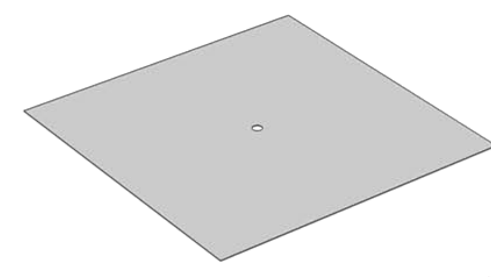

b)

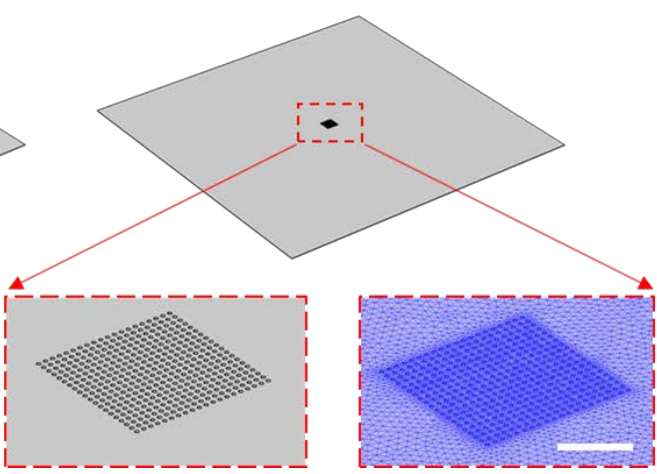

Figure 3. Computer-aided design (CAD) models used for the finite element method (FEM)-based simulations. (a) CAD model used for linear elastic simulations. (b) CAD model used for the nonlinear elastic simulations. A detail of the nanoapertures in the CAD model and the corresponding mesh are shown as insets. Scale bar represents $10 \mu \mathrm{m}$.

Figure 4 shows images of selected values for the deflection and stress distribution of the model of the membrane under an applied pressure of 20 psi. Figure $4 \mathrm{a}, \mathrm{b}$ show the displacement in the $z$-direction for the linear and nonlinear models, respectively. The results are presented as non-deformed, with vectors representing the direction and magnitude of the deflection. The pattern of deflection observed from the simulations, as expected, is quasi-circular, with increasing magnitude toward the center of the free-standing membrane. The maximum deflection values, for the linear and nonlinear 
simulations at an applied pressure of 20 psi, were 24.08 and $19.39 \mu \mathrm{m}$, respectively. Maxima were always obtained at the apex of the deformed membrane. Figure $4 \mathrm{c}, \mathrm{d}$ show the von Mises stress distribution for an applied pressure of 20 psi. The maximum stress found in the simulations was on the order of $1 \times 10^{8}$ to $10 \times 10^{8} \mathrm{~Pa}$, which suggests that the substrate which is housing the nanoapertures could adequately withstand the deformations resulting from the applied pressure. The simulation results were used to define a range of pressure that could be used experimentally, avoiding failure of the membrane.

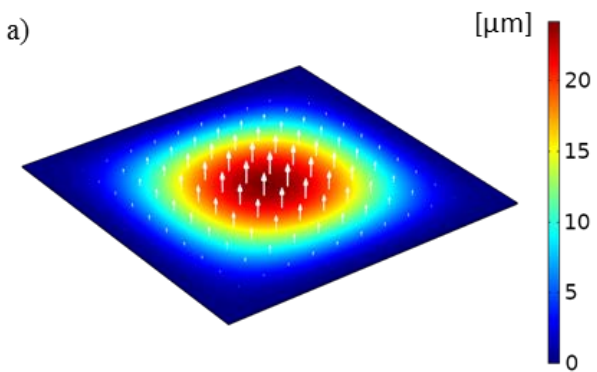

c)

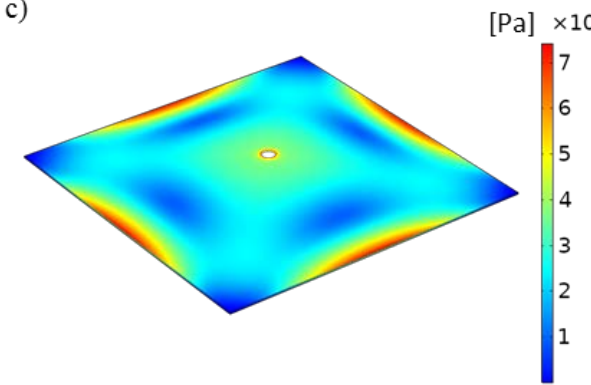

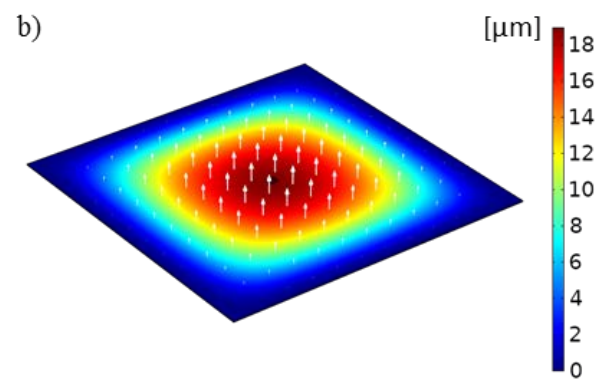

d)

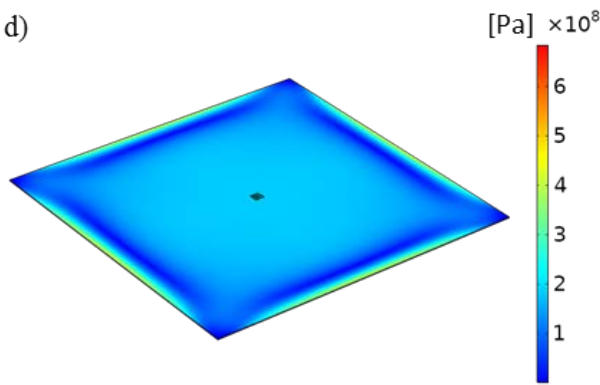

Figure 4. Simulation results of linear and nonlinear models. Membrane deflection under an applied pressure of 20 psi for (a) the linear model and (b) the nonlinear model. The apertures are shown in the insets within yellow dashed boxes in both cases. Stress distribution (von Mises yield criterion) under an applied pressure of 20 psi for (c) the linear model and (d) the nonlinear model.

Figure 5 shows a bright-field microscopy image of the Au-on-nitride membrane before and after the application of a pressure of 10 psi. The substrate included two rectangular periodic arrays of nanoapertures, indicated with yellow dashed lines. The boundaries of the $\mathrm{Si}_{3} \mathrm{~N}_{4}$ membrane are indicated by red dashed lines. The focal plane in both images is the same, which indicates the deflection of the substrate under the applied pressure.
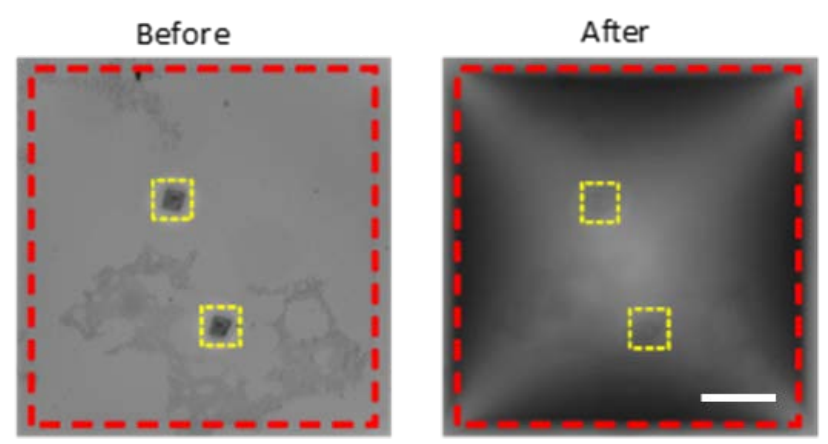

Figure 5. Membrane deflection before and after the application of a pressure of 20 psi. Scale bar represents $100 \mu \mathrm{m}$. 
In order to measure the deflection experimentally, the elevation difference at the apex of the membrane was used as reference, and the in-focus $z$-positions were recorded. The applied pressure on the surface of the substrate was monitored and regulated to achieve a constant value throughout the measurement of the deflection. Fringe patterns can be observed in the deflected membrane case, which correspond to the reflected light, confirming a level gradient along the surface of the substrate, and a maximum translation at the apex. The $z$-positioning precision of the inverted microscopy system used in this study was $0.2 \mu \mathrm{m}$, which allowed measuring deflections with micrometer precision, at $2-\mu \mathrm{m}$ intervals.

Figure 6 shows experimental and simulations results for applied pressures of $1-20$ psi. The trend from the linear simulation model was linear, as expected, with corresponding minimum and maximum deflections of 1.209 and $24.08 \mu \mathrm{m}$. In contrast, the deflection results from the nonlinear model decreased with the applied pressure, with minimum and maximum values of 2.584 and $19.39 \mu \mathrm{m}$. The same trend was found for experimental values, with the magnitude of the maximum deflection at the apex decreasing with the applied pressure. This can be explained by considering the physical restriction along the frame of the free-standing membrane and due to the mechanical properties of the material. The figure also shows the results from three analytical models that were used to obtain theoretical values, i.e., the Rijn et al. [22], Ugural [28], and Kovacs et al. [29] models, as well as an adjusted Kovacs model fit with the experimental values. These models are similar to each other, whereby they all consider the perforation in a membrane as an error factor affecting the Young's modulus of the membrane. The deflection of a membrane is given by Equation (1) [30].

$$
w_{\max }=k_{0} L \sqrt[3]{\frac{P_{0} L}{E_{\text {eff }} h^{\prime}}}
$$

where $w$ is the z-axis displacement, $L$ and $h$ are the size and the thickness of the membrane, and $P_{0}$ is the applied pressure. The constant $k_{0}$ is equal to $0.318,0.325$, and 0.319 within Rijn's, Ugural's, and Kovacs' models, respectively [30]. $E_{\text {eff }}$ is the effective Young's modulus, calculated as $E_{\text {eff }}=(1-P) E_{\text {closed }}$, where $E_{\text {closed }}$ is the Young's modulus of unperforated membrane, and $P$ is the correction factor. $P$ is dependent on the perforation and is defined as the fraction of the open areas over the total area of the membrane. As the models are similar, there is negligible difference between the deflection values obtained using the three different models [30]. In the case of the optofluidic sensor, the deformable section of the membrane is smaller than the $500 \mu \mathrm{m}$ by $500 \mu \mathrm{m}$ of the free-standing substrate, as observed in Figure 5. The theoretical models do not consider the frame around the deformable area. Therefore, there is an offset between the deflection values obtained using the models and those obtained experimentally, as shown in Figure 6. 


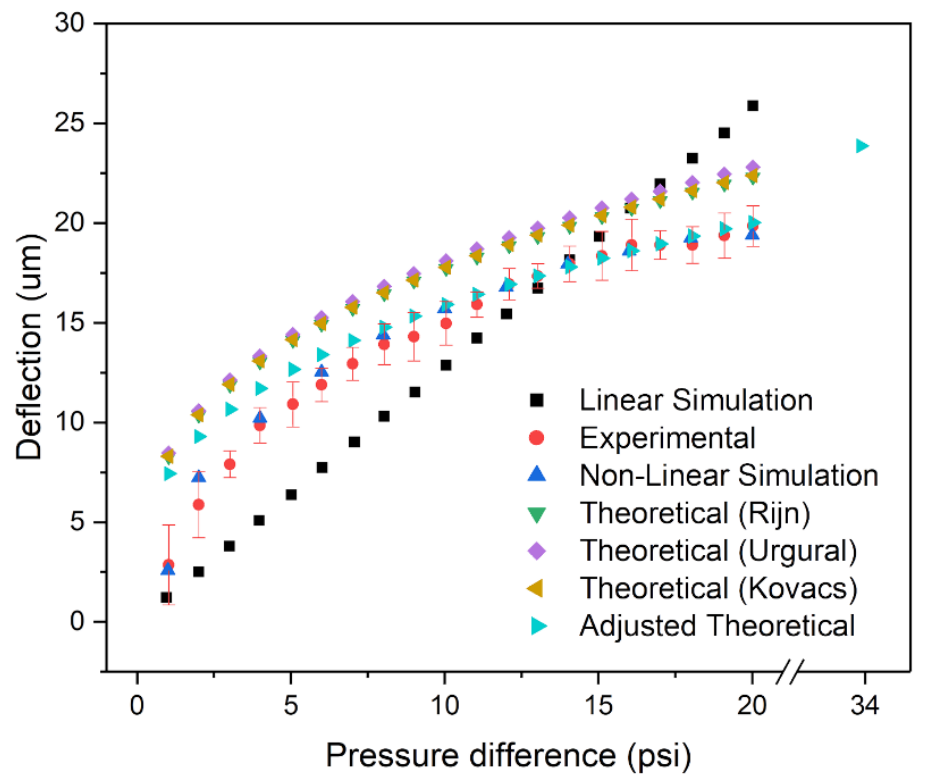

Figure 6. Experimental, theoretical, and simulation results of the maximum membrane deflection (apex). Error bars indicate standard deviation $(n=5)$.

The experimental results have a similar trend compared to the theoretical models. Over the non-linear region $(<7 \mathrm{psi})$, the experimental values are on average $\sim 32 \%$ below the theoretical maximum, and $\sim 12 \%$ below the theoretical maximum within the linear region ( $7 \mathrm{psi}$ ). The slopes for the experimental and theoretical (Kovacs) values were 0.4831 and $0.4826 \mu \mathrm{m} / \mathrm{psi}$, respectively within the linear region, with $R^{2}$ (coefficient of determination (COD)) values of 0.937 and 0.993 , respectively. The slopes indicate that the models do not quantify the actual deflection of the membrane. However, they accurately represent the trend of the membrane's deflection. As such, the unperforated area around the nanohole arrays is influential on the mechanical stability of the membrane. With the assumption that some length of area around the unperforated area does not deflect, then the deflection of the membrane can be rewritten as follows:

$$
w_{\text {max }}=k_{0} L_{e f f} \sqrt[3]{\frac{P_{0} L_{e f f}}{E_{e f f} h}}
$$

where $L_{e f f}$, is the effective length of the membrane based on the experimental values, calculated as $L_{e f f}=\sqrt{\text { Area of holes } / P_{e f f}} . P_{e f f}$ is the effective correction factor based on the experimental values, where it is assumed that some length around the unperforated area does not deflect. $E_{e f f}$ is adjusted to the experimental values and calculated as $E_{e f f}=\left(1-P_{e f f}\right) E_{\text {closed }}$. The model found a range of $P_{e f f}$ values based on each experimental deflection point from $2.138 \times 10^{-3}$ to $5.09 \times 10^{-4}$, corresponding to effective membrane lengths of $225 \mu \mathrm{m}$ to $460 \mu \mathrm{m}$, respectively. Figure 6 illustrates that the model is incapable of fitting all the experimental values with one value of $P_{\text {eff }}$. The initial deflection value of the experimental values has an $L_{e f f}$ of $225 \mu \mathrm{m}$, where the $L_{e f f}$ non-linearly increases until it plateaus to a constant value of $460 \mu \mathrm{m}$ within the linear region of the experimental values. The effective length paints a clear image of the membrane's behavior under pressure. Initially, at low pressures, only the center area of the membrane deflects, while the majority of the membrane is not affected by the applied pressures. As the applied pressure increases, the deflected area grows until it reaches a maximum constant value $(460 \mu \mathrm{m})$. Even at the maximum value of effective lengths, some outer areas of the membrane do not deflect, reassuring the limitations of deflection model. The experiment was not designed to bring the substrate to mechanical failure; however, the pressure value for the breaking point can be extrapolated from the theoretical model based on the material's properties. The inflection 
point of the membrane is not at the edges of the membrane but limited to the effective length of the membrane (i.e., $L_{e f f}$ ). Based on the Rijn et al. and Timoshenko et al. models, the maximum pressure applied can be found based on the total stress of the material as shown in Equation (3) [22,31].

$$
\sigma_{\text {total }}=\sigma_{\text {tensile }}+\sigma_{\text {bend }}=\frac{0.297}{1-v}\left(1+\frac{1.439}{0.358}\right) \sqrt[3]{\frac{P_{0}^{2} L_{e f f}^{2} E_{e f f}}{\left(1-v^{2}\right) h^{2}}}
$$

where $\sigma_{\text {total }}$ is the total stress of the membrane, and $\sigma_{\text {tensile }}$ and $\sigma_{\text {bend }}$ are the tensile stress due to stretching and the maximum bending stress near the middle of the membrane's deflection edges, respectively. The model is valid when the substrate is under a substantial load that results in large deflections (i.e., $w_{\max } / h \gg 1$ ). Considering that the reported ultimate stress, $\sigma_{\text {ultimate }}$, is on the order of $10^{9} \mathrm{~Pa}$, and the intrinsic tensile stress is $10^{8} \mathrm{~Pa}$ for a silicon nitride membrane, then the internal stresses can be neglected since they are an order of magnitude lower than the total stress [22]. For a nonductile inorganic material, the $\sigma_{\text {ultimate }}$ is equivalent to its yield stress. Taking $2.5 \mathrm{GPa}$ as $\sigma_{\text {total }}$, based on the mechanical properties of the material, a pressure of 33.91 psi and deflection of $23.87 \mu \mathrm{m}$ are obtained, corresponding to the maximum possible values at the verge of mechanical failure [32]. This theoretical maximum deflection value at the verge of failure, which adequately follows the trend of the adjusted theoretical curve, is shown in Figure 6.

\section{Conclusions}

This work presented an investigation of the deflection and structural stability of optofluidic nanohole array-based sensors operating in flow-through mode. The study was approached using experiments, theoretical models, and FEA via computer simulations through FEM. Linear and nonlinear material models were simulated using COMSOL Multiphysics software. The simplified linear model had an expected discrepancy with experimental values, but these were useful to obtain an estimation of the order of magnitude of transmembrane pressures that would allow studying the deflection of the substrate when used in flow-through operation, while avoiding mechanical failure. The discrepancies were up to $\sim 20 \%$. In contrast, the nonlinear model, accounting for a complete nanohole array, accurately described the deflection values obtained experimentally. The stresses corresponding to these deflections can be used to predict maximum operation values that could prevent failure of the optofluidic nanostructures. Three analytical models were used to analyze the deformation of the sensor. The models depicted the behavior of the deflected substrate under pressure but did not intrinsically fit the experimental results since only a fraction of the surface deflects due to the attachment of the free-standing substrate to the silicon frame. Even when the entire $500-\mu \mathrm{m}$ membrane is under pressure, only a reduced square area, ranging from $225 \mu \mathrm{m}$ to a maximum of $460 \mu \mathrm{m}$ per side, deflects. Once adjusted, the theoretical model better fit the experimental deflection values. Based on the models, the fracture point was extrapolated from the maximum yield stress of silicon nitride membranes. As the membranes are composed of nonductile, inorganic material, their yield stress is equivalent their ultimate stress, which resulted with a maximum possible deflection of $23.9 \mu \mathrm{m}$, with the applied pressure of 33.9 psi. Although the optofluidic structures are limited by their fragile mechanical stability in flow-through operation, these results show that they are capable of withstanding transmembrane pressures compatible with sensing applications, where the analyte is required to be brought into the apertures. Simulations that could predict the deflection of the structures would greatly benefit the design needs of flow-through optofluidic platforms for specific applications in the context of biosensing.

Author Contributions: Conceptualization, C.E.; methodology, Y.B., J.G.-C., C.E.; software, Y.B., J.G.-C., C.E.; validation, C.E.; formal analysis, Y.B., J.G.-C., C.E.; investigation, Y.B., J.G.-C., C.E.; resources, C.E.; data curation, C.E.; writing-original draft preparation, Y.B., J.G.-C., C.E.; writing-review and editing, C.E.; supervision, C.E.; project administration, C.E.; funding acquisition, C.E. All authors have read and agreed to the published version of the manuscript.

Funding: C.E. acknowledges funding from the Natural Sciences and Engineering Research Council of Canada (NSERC) RGPIN-2010-5138, and Canada Foundation for Innovation John R. Evans Leaders' Fund Program 
(No. 319670). Y.B. acknowledges funding from Materials for Advanced Photonics and Sensing (MAPS) funded through NSERC CREATE and Ontario Graduate Scholarship (OGS). J.G.-C. acknowledges the national graduate scholarship provided by the National Council for Science and Technology of Mexico (CONACYT).

Conflicts of Interest: The authors declare no conflicts of interest.

\section{References}

1. Marcoux, R.M.; Vogenberg, F.R. Telehealth: Applications From a Legal and Regulatory Perspective. P T Peer-Rev. J. Formul. Manag. 2016, 41, 567-570.

2. Preechaburana, P.; Gonzalez, M.C.; Suska, A.; Filippini, D. Surface Plasmon Resonance Chemical Sensing on Cell Phones. Angew. Chem. Int. Ed. 2012, 51, 11585-11588. [CrossRef]

3. Comina, G.; Suska, A.; Filippini, D. Autonomous chemical sensing interface for universal cell phone readout. Angew. Chem. Int. Ed. 2015, 54, 8708-8712. [CrossRef]

4. Srinivasan, B.; Tung, S. Development and applications of portable biosensors. J. Lab. Autom. 2015, 20, 365-389. [CrossRef]

5. Li, G.; De Clercq, E. Therapeutic options for the 2019 novel coronavirus (2019-nCoV); Nature Publishing Group: New York, NY, USA, 2020.

6. Boratto, P.V.; Oliveira, G.P.; Machado, T.B.; Andrade, A.C.S.; Baudoin, J.-P.; Klose, T.; Schulz, F.; Azza, S.; Decloquement, P.; Chabriere, E.; et al. A mysterious $80 \mathrm{~nm}$ amoeba virus with a near-complete" ORFan genome" challenges the classification of DNA viruses. bioRxiv 2020. [CrossRef]

7. Brolo, A.G.; Gordon, R.; Leathem, B.; Kavanagh, K.L. Surface plasmon sensor based on the enhanced light transmission through arrays of nanoholes in gold films. Langmuir 2004, 20, 4813-4815. [CrossRef]

8. Ebbesen, T.W.; Lezec, H.J.; Ghaemi, H.F.; Thio, T.; Wolff, P.A. Extraordinary optical transmission through sub-wavelength hole arrays. Nature 1998, 391, 667-669. [CrossRef]

9. Craighead, H. Future lab-on-a-chip technologies for interrogating individual molecules. In Nanoscience And Technology: A Collection of Reviews from Nature Journals; World Scientific: Singapore, Singapore, 2010; pp. 330-336.

10. Ahn, C.H.; Choi, J.-W.; Beaucage, G.; Nevin, J.H.; Lee, J.-B.; Puntambekar, A.; Lee, J.Y. Disposable smart lab on a chip for point-of-care clinical diagnostics. Proc. IEEE 2004, 92, 154-173. [CrossRef]

11. Herrmann, M.; Veres, T.; Tabrizian, M. Enzymatically-generated fluorescent detection in micro-channels with internal magnetic mixing for the development of parallel microfluidic ELISA. Lab Chip 2006, 6, 555-560. [CrossRef]

12. Chin, C.D.; Linder, V.; Sia, S.K. Lab-on-a-chip devices for global health: Past studies and future opportunities. Lab Chip 2007, 7, 41-57. [CrossRef]

13. Gomez-Cruz, J.; Nair, S.; Ascanio, G.; Escobedo, C. Flow-through nanohole array based sensor implemented on analogue smartphone components. In Proceedings of the SPIE Nanoscience + Engineering, San Diego, CA, USA, 25 August 2017.

14. Soler, M.; Belushkin, A.; Cavallini, A.; Kebbi-Beghdadi, C.; Greub, G.; Altug, H. Multiplexed nanoplasmonic biosensor for one-step simultaneous detection of Chlamydia trachomatis and Neisseria gonorrhoeae in urine. Biosens. Bioelectron. 2017, 94, 560-567. [CrossRef] [PubMed]

15. Yanik, A.A.; Huang, M.; Kamohara, O.; Artar, A.; Geisbert, T.W.; Connor, J.H.; Altug, H. An Optofluidic Nanoplasmonic Biosensor for Direct Detection of Live Viruses from Biological Media. Nano Lett. 2010, 10, 4962-4969. [CrossRef]

16. Escobedo, C.; Chou, Y.W.; Rahman, M.; Duan, X.B.; Gordon, R.; Sinton, D.; Brolo, A.G.; Ferreira, J. Quantification of ovarian cancer markers with integrated microfluidic concentration gradient and imaging nanohole surface plasmon resonance. Analyst 2013, 138, 1450-1458. [CrossRef]

17. Gomez-Cruz, J.; Nair, S.; Manjarrez-Hernandez, A.; Gavilanes-Parra, S.; Ascanio, G.; Escobedo, C. Cost-effective flow-through nanohole array-based biosensing platform for the label-free detection of uropathogenic E. coli in real time. Biosens. Bioelectron. 2018, 106, 105-110. [CrossRef]

18. Escobedo, C.; Brolo, A.G.; Gordon, R.; Sinton, D. Optofluidic concentration: Plasmonic nanostructure as concentrator and sensor. Nano Lett. 2012, 12, 1592-1596. [CrossRef] [PubMed]

19. Cruz, S.; Hönig-d'Orville, A.; Müller, J. Fabrication and optimization of porous silicon substrates for diffusion membrane applications. J. Electrochem. Soc. 2005, 152, C418-C424. [CrossRef] 
20. Sinton, D.; Gordon, R.; Brolo, A.G. Nanohole arrays in metal films as optofluidic elements: Progress and potential. Microfluid. Nanofluidics 2008, 4, 107-116. [CrossRef]

21. Wu, H.; Liu, G.; Wang, J. Atomistic and continuum simulation on extension behaviour of single crystal with nano-holes. Model. Simul. Mater. Sci. Eng. 2004, 12, 225. [CrossRef]

22. Van Rijn, C.; van der Wekken, M.; Nijdam, W.; Elwenspoek, M. Deflection and maximum load of microfiltration membrane sieves made with silicon micromachining. J. Microelectromech. Syst. 1997, 6, 48-54. [CrossRef]

23. Escobedo, C.; Brolo, A.G.; Gordon, R.; Sinton, D. Nanoplasmonics as nanofluidics: Transport and sensing in flowthrough nanohole arrays. In Proceedings of the Microfluidics, BioMEMS, and Medical Microsystems IX, San Francisco, CA, USA, 14 February 2011.

24. Escobedo, C. On-chip nanohole array based sensing: A review. Lab Chip 2013, 13, 2445-2463. [CrossRef]

25. Duffy, D.C.; McDonald, J.C.; Schueller, O.J.A.; Whitesides, G.M. Rapid prototyping of microfluidic systems in poly(dimethylsiloxane). Anal. Chem. 1998, 70, 4974-4984. [CrossRef] [PubMed]

26. Luecke, W.E.; Wiederhorn, S.M. A new model for tensile creep of silicon nitride. J. Am. Ceram. Soc. 1999, 82, 2769-2778. [CrossRef]

27. Wunderlich, W. Ceramic Materials; InTech: Rijeka, Croatia, 2010.

28. Ugural, A.C. Stresses in Beams, Plates, and Shells; CRC press: Boca Raton, FL, USA, 2009.

29. Kovács, Á.; Vízváry, Z.; Kovács, A.; Mescheder, U. Large deflection analysis of perforated silicon nitride membranes. In Proceedings of the 6th ESMC, Budapest, Hungary, 28 August-1 September 2006.

30. Kovacs, A.; Kovács, Á.; Pogány, M.; Mescheder, U. Mechanical investigation of perforated and porous membranes for micro-and nanofilter applications. Sens. Actuators B Chem. 2007, 127, 120-125. [CrossRef]

31. Timoshenko, S.P.; Woinowsky-Krieger, S. Theory of Plates and Shells; McGraw-hill: New York, NY, USA, 1959.

32. Boé, A.; Coulombier, M.; Safi, A.; Pardoen, T.; Raskin, J.-P. On-chip Testing Laboratory for Nanomechanical Characterization of Thin Films. In Proceedings of the 2009 SEM Annual Conference and Exposition on Experimental and Applied Mechanics, Albuquerque, NM, USA, 1-4 June 2009.

(C) 2020 by the authors. Licensee MDPI, Basel, Switzerland. This article is an open access article distributed under the terms and conditions of the Creative Commons Attribution (CC BY) license (http://creativecommons.org/licenses/by/4.0/). 